\title{
PESSOAS EM SITUAÇÃO DE RUA: REVISÃO SISTEMÁTICA
}

Gesyele Batista Oliveira

Universidade Tuiuti do Paraná

Débora Mayumi Higa

Universidade Tuiuti do Paraná

Jackeline Claudino Sousa Araujo

Universidade Tuiuti do Paraná

Aline Moraes Selusnhaki

Universidade Tuiuti do Paraná

Tomás Collodel Magalhães Reis

Universidade Tuiuti do Paraná

Adriano Valério dos Santos Azevêdo

Universidade Tuiuti do Paraná
Recebido em: 25/07/2019

$1^{\text {a }}$ revisão em: $18 / 11 / 2019$

Aceito em: 25/02/2020

\section{RESUMO}

O presente artigo objetivou realizar uma revisão sistemática das pesquisas empíricas sobre pessoas em situação de rua. Foram consultadas as bases de dados (Lilacs, Medline, Ibecs, Redalyc, PePSIC) para identificar as pesquisas publicadas no período de 2011 a 2016. Identificaram-se 23 artigos que foram categorizados: 1. Aspectos sociodemográficos e macro determinantes; 2 . Processo saúde e doença; 3. Modos de vida e significações e 4. Identidade social. Destacou-se o aumento da população em situação de rua e aspectos da vulnerabilidade social: uso de álcool e drogas, relatos de violência, o cotidiano de morar nas ruas, a discriminação, a fragilização de vínculos familiares, e as transformações nas identidades que repercutem na exclusão social. Trata-se de uma temática de estudo que possibilita a análise de aspectos psicossociais para o desenvolvimento de projetos de intervenção voltados para a integralidade do cuidado.

Palavras-chave: pessoas em situação de rua; exclusão social; vulnerabilidade social 


\section{HOMELESS PEOPLE: SYSTEMATIC REVIEW}

\section{ABSTRACT}

This article aimed to conduct a systematic review of the empirical research on homeless people. Lilacs, Medline, Ibecs, Redalyc, and PePSIC databases were searched to identify research studies published between 2011 and 2016.Twentythree articles were found and categorized as: 1 . Sociodemographic aspects and macro determinants; 2. Health and disease processes; 3. Lifestyles and significations; and 4. Social identities. An increase in the homeless population was found, and the following aspects of social vulnerability were identified: alcohol and drug use, reports of violence, the daily life in the streets, prejudice, weakening of family bonds, and identity changes that influence social exclusion. This study theme enables an analysis of psychosocial aspects for the development of intervention projects aimed at integral care.

Keywords: homeless people; social exclusion; social vulnerability.

\section{PERSONAS SIN HOGAR: REVISIÓN SISTEMÁTICA}

\section{RESUMEN}

O presente artículo objetivo realizar una revisión sistemática de la investigación empírica en personas sin hogar. Las bases de datos (Lilacs, Medline, Ibecs, Redalyc, PePSIC) fueron consultadas para identificar la investigación publicada de 2011 a 2016. Fueron identificados y categorizados 23 artículos: 1. Aspectos sociodemográficos y macro determinantes; 2 . Proceso de salud y enfermedad; 3. Formas de vida y significados y 4. Identidad social. Se destacó el aumento de la población sin hogar y los aspectos de vulnerabilidad social: consumo de alcohol y drogas, relatos de violencia, vida cotidiana en la calle, discriminación, fragilidad de los lazos familiares y cambios en las identidades que reflejan en exclusión social. Este es un tema de estudio que permite el análisis de los aspectos psicosociales para el desarrollo de proyectos de intervención dirigidos a la atención integral.

Palabras clave: personas en situación de calle; exclusión social; vulnerabilidad social. 


\section{INTRODUÇÃO}

A população em situação de rua representa um grupo populacional heterogêneo que vive em condições de pobreza extrema, não possui moradia convencional regular e os vínculos com a família foram rompidos ou fragilizados (Ministério da Saúde, 2008). Em 2014, o Movimento Nacional de População de Rua estimou a presença de 50 mil pessoas em situação de rua (Ministério da Saúde, 2014). O Instituto de Pesquisas Econômicas Aplicadas (IPEA) realizou em 2015 uma estimativa numérica com base nos dados coletados em 1.924 municípios por meio do Censo do Sistema Único de Assistência Social (Ministério do Planejamento e Gestão, 2016). E por meio de um modelo teórico que considera variáveis, tais como, crescimento demográfico e vulnerabilidade social, o IPEA (2016) estimou a existência de 101.854 pessoas em situação de rua no Brasil (Ministério do Planejamento e Gestão, 2016). Apesar das divergências em relação ao número de pessoas em situação de rua, no âmbito nacional é crescente o aumento dessa população, o que repercute em desigualdade social e demandas a serem atendidas por meio de políticas públicas e ações comunitárias.

De acordo com as Orientações técnicas do Centro de Referência Especializado para População de rua e Serviço Especializado para pessoas em situação de rua (Ministério do Desenvolvimento Social e Combate à Fome, 2014), uma parte destas pessoas possuem redes de apoio pré-estabelecidas que os auxiliam nos momentos de dificuldades. No entanto, com base neste documento, também existem pessoas que possuem menores possibilidades de auxílio por parte de conhecidos, amigos ou parentes, e, portanto, estabelecem um vínculo com a rua para a criação de mecanismos de sobrevivência. Consolidada a vinculação na situação de rua, as redes de apoio auxiliam nas relações pessoais, mas por outro lado, existe a falta de acesso a serviços públicos (Ministério do Desenvolvimento Social e Combate à Fome, 2014). Partindo deste entendimento de como ocorre à vinculação das pessoas no ambiente da rua, podemos compreender melhor as especificidades e vulnerabilidades sociais que estes vivenciam.

A vulnerabilidade refere-se à falta de acesso aos direitos que são oferecidos pelo estado, mercado e sociedade. Estes direitos incluem: bem-estar, moradia, trabalho, renda, saúde, educação, serviços públicos, vínculos afetivos e acesso à informação (Presidência da República, 2009). Assim, a população em situação de rua vive em situação de vulnerabilidade social por não possuir moradia convencional e apresentar dificuldade de acesso aos serviços de saúde (Grangeiro et al., 2012), e outros setores das políticas públicas devido as condições de pobreza extrema. Desta forma, no contexto brasileiro é necessário desenvolver ações intersetoriais para fomentar a promoção da saúde com o objetivo de minimizar as vulnerabilidades sociais.

Com a presença de um número expressivo de pessoas vivendo nas ruas do território brasileiro e com a recente criação das políticas públicas voltadas para a população de rua (Presidência da República, 2009), trabalhos científicos, 
especificamente revisões sistemáticas investigaram o contexto de crianças e adolescentes (Herrera, Rojas, \& Jiménez, 2016), de mães adolescentes grávidas (Scappaticci \& Blay, 2010), e de idosos (Falcão et al., 2012). Esses estudos indicam a necessidade de construção e efetivação de trabalhos preventivos para resgatar a cidadania e promover inclusão social.

Percebe-se a escassez de estudos de revisão sistemática, assim, verifica-se a necessidade de um levantamento da produção científica para fins de caracterizar as temáticas e contextualizá-las com o propósito de refletir sobre estratégias de intervenção de maneira intersetorial. A realização deste levantamento possibilita que as equipes da assistência social e da saúde possam ter acesso a um conteúdo científico que oportunize acesso aos conhecimentos para atender as especificidades deste grupo populacional. No campo científico, permite a apresentação e discussão dos estudos para que isto viabilize reflexões para novas pesquisas que possam avaliar programas de intervenção e gerar tecnologias e práticas sociais de inclusão. O presente artigo objetivou realizar uma revisão sistemática dos estudos empíricos sobre pessoas em situação de rua.

\section{MÉTODO}

\section{DELINEAMENTO}

Realizar uma revisão sistemática implica em investigar e integrar um conjunto de estudos que possuem resultados semelhantes ou divergentes, para que isso possibilite identificar possíveis lacunas de conhecimentos para futuras investigações (Sampaio \& Mancini, 2007).

Inicialmente foi realizada uma consulta nos Descritores em Ciências da Saúde (DECS) para identificar a terminologia e agilizar o processo de buscas nas bases de dados. O DECS é um vocabulário controlado, estruturado e trilíngue criado pela Bireme (Centro Latino-Americano e do Caribe de Informação em Ciências da Saúde) para utilização na indexação de artigos de revistas científicas, anais e outros tipos de materiais, assim como para ser usado na pesquisa e recuperação de assuntos da literatura científica nas bases de dados. Foram identificados os seguintes descritores: pessoas em situação de rua/ personas sin hogar. No próximo passo foram iniciadas as consultas com estes descritores nas bases de dados: Lilacs, Medline, Ibecs, Redalyc, PePSIC.

\section{CRITÉRIOS DE INCLUSÃO E EXCLUSÃO}

Procurou-se incluir os estudos realizados nos anos de 2011-2016 publicados em português e espanhol nos países da América Latina e Europa. Buscou-se incluir as pesquisas empíricas nas quais as pessoas em situação de rua foram participantes e responderam algum instrumento de pesquisa. Foram incluídos os estudos que disponibilizaram o texto completo, e excluídos os estudos teóricos e de revisão. 


\section{PROCEDIMENTOS}

Logo após a extração dos artigos das bases de dados, foram aplicados os critérios de inclusão/exclusão e a retirada de estudos duplicados, posteriormente utilizouse uma planilha para colocar as seguintes informações: nome dos autores, ano de publicação, resumo, objetivo, base de dados, síntese dos resultados obtidos e periódico no qual o estudo foi publicado. Utilizou-se a recomendação internacional - Preferred Reporting Items for Systematic Reviews and Meta-Analyses (PRISMA disponível em: www.prisma-statement.org) - no processo de identificação, seleção e elegibilidade dos artigos com o propósito de atender critérios de qualidade.

\section{ANÁLISE DE DADOS}

Realizou-se a leitura do título e objetivo dos artigos para a categorização temática, assim, utilizou-se a análise temática para a construção de categorias (Bardin, 2002). $\mathrm{Na}$ próxima etapa, ocorreu a leitura integral dos artigos para a escrita de resultados.

\section{RESULTADOS}

Na Figura 1 é possível verificar o fluxograma do processo de busca, seleção e inclusão de artigos. Dos 23 artigos científicos, 16 foram publicados em revistas nacionais: Psicologia: Ciência e Profissão (1), Revista Saúde Pública (1), Caderno Saúde Pública (1), Psicologia em Estudo (1), Psicologia e Saber Social (1), Psicologia: Reflexão e Crítica (1), Sociedade e Cultura (1), Saúde e Sociedade (5), Interface, comunicação e saúde (1), Fractal Revista de Psicologia (1), REME: Revista Mineira de Enfermagem (1), Revista da Escola de Enfermagem da USP (1). Sete artigos publicados em revistas internacionais: Anales de Psicología (2), Revista Costarr Salud Pública (1), Revista Colombiana de Psicología (1), Revista Chilena de Terapia Ocupacional (1), Psicología para América Latina (1), Revista Peruana de Medicina Experimental y Salud Publica (1). Referente ao ano de publicação e frequência dos estudos: 2011 (2), 2012 (2), 2013 (5), 2014 (6), 2015 (7) e 2016 (1).

Em relação à metodologia das pesquisas consultadas, oito estudos quantitativos e quinze artigos qualitativos. Sobre os participantes dos estudos, foi possível identificar adolescentes, de ambos os sexos, homens, mulheres e gestantes, entretanto, ocorreu a prevalência do gênero masculino nos estudos. Depois de realizada a leitura das pesquisas, foi necessário dividir os estudos em categorias: 1 . Aspectos sociodemográficos e macro determinantes, 2. Processo saúde-doença, 3. Modos de vida e significações, e 4. Identidade social. 


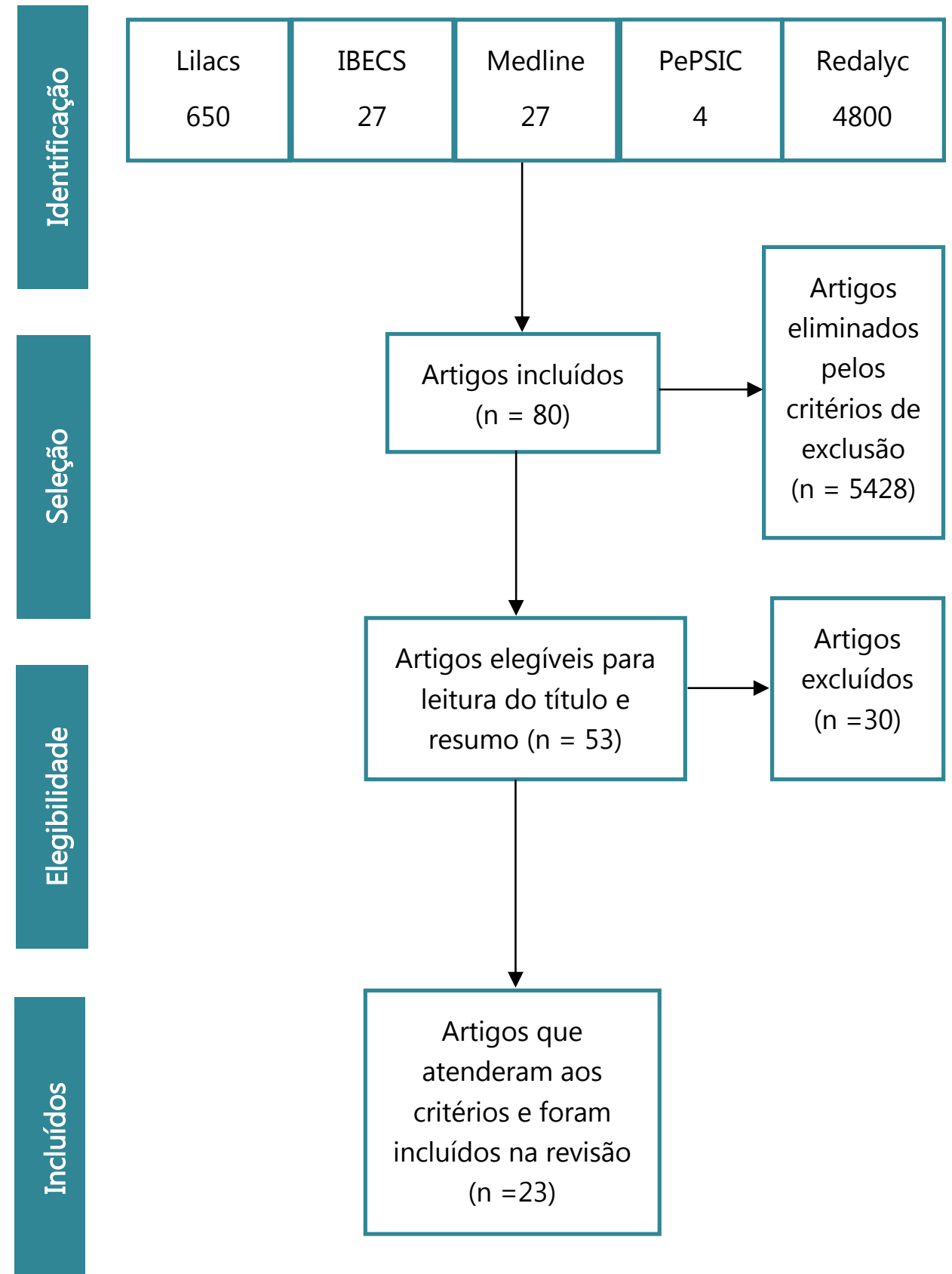

Figura 1. Fluxograma do processo de busca e seleção de artigos.

\section{ASPECTOS SOCIODEMOGRÁFICOS E MACRO DETERMINANTES}

Um estudo foi realizado com 251 indivíduos para verificar aspectos sociodemográficos e os resultados apontaram a predominância de adultos do sexo masculino, negros, com baixo nível de escolaridade, renda mensal menor que meio salário mínimo, exercendo trabalhos sem vínculo empregatício (Barata, CarneiroJúnior, Ribeiro, \& Silveira, 2015). Alguns indivíduos estavam vivendo na rua há mais 
de cinco anos ( $n=81 / 33,2 \%)$, e outros há menos de um ano $(n=76 / 31,1 \%)$. Dos indivíduos que possuíam família identificou-se que estavam separados por escolha pessoal ( $n=53 / 30,1 \%$ ) ou motivos não especificados ( $n=61 / 34,7 \%)$, e os outros não tinham ajuda de amigos ou parentes. Foram identificados os motivos que levaram à situação de rua: condições financeiras precárias $(n=158 / 62,9 \%)$ e ausência da família ( $n=81 / 32,3 \%$ ), por outro lado, relataram o desejo de sair dessa situação em virtude da discriminação vivenciada ( $n=212 / 85,8 \%$ ). Os tipos de agressões sofridas foram as seguintes: físicas ou sexuais, sendo algumas realizadas por policiais. Dentre as situações de risco, o fumo, o consumo de álcool e drogas. Tais aspectos se referem as vulnerabilidades vivenciadas pelas pessoas em situação de rua, pelo fato de que a exposição aos riscos representa um fator agravante para a exclusão social.

Ferreira (2011) desenvolveu um estudo com crianças e adolescentes, até 18 anos de idade incompletos, que estavam exercendo qualquer tipo de atividade nas ruas por uma semana. Os resultados referentes à escolarização demonstraram que se diminuída a evasão escolar haveria uma redução do número de crianças nas ruas. Identificou-se a média de crianças e adolescentes nas ruas frequentando a escola ( $n=2.285 / 75,66 \%)$, e o fato que levou esses indivíduos a situação de rua foi a convivência em comunidades e famílias pobres, e por isso buscaram nas ruas diversas soluções para o enfrentamento da vulnerabilidade social.

Numa pesquisa com pessoas maiores de 60 anos verificou-se que poucos possuíam familiares próximos, e em relação à escolaridade, alguns tinham ensino fundamental completo ou incompleto, enquanto outros não tinham instrução escolar básica (Risco, Léon, Dongo, \& Munayco, 2015). Foram identificadas doenças mentais, crônicas e problemas com álcool e uso de drogas. De acordo com a análise sociofamiliar, verificou-se que se encontravam em risco social por não possuírem apoio familiar e uma rede de suporte social.

\section{PROCESSO SAÚDE-DOENÇA}

\section{TRANSTORNOS MENTAIS E DE PERSONALIDADE}

Salavera (2014) investigou se os transtornos mentais e de personalidade eram as causas das desistências aos tratamentos psicológicos e de inserção social de pessoas em situação de rua. Os resultados identificaram que as pessoas com depressão maior e àqueles com transtornos psicóticos não frequentam os centros de atendimentos psicossociais, visto que, as condições cognitivas geralmente se encontram prejudicadas. $\mathrm{E}$ as pessoas com transtornos de personalidade são mais propensas ao abandono dos recursos terapêuticos e sociais.

Aspectos de personalidades patológicas entre os moradores de rua num grupo de pacientes psiquiátricos foram analisados (Montiel, Bartholomeu, Carvalho, \& Pessoto, 2015). Os resultados evidenciaram que a ausência de tratamento adequado e acompanhamento com profissionais especializados comprometem a saúde destas pessoas, além disso, a história de vida de cada indivíduo e o ambiente 
hostil no qual estão inseridos representa um agravante para o desenvolvimento de patologias mentais. Os autores identificaram os transtornos de personalidade mais prevalentes: paranoide, esquizotípico, antissocial e histriônico.

\section{VULNERABILIDADE ÀS DOENÇAS SEXUALMENTE TRANSMISSÍVEIS}

A pesquisa de Grangeiro et al. (2012) objetivou analisar a prevalência e a caracterização de vulnerabilidade ao Vírus da Imunodeficiência Humana (HIV) dos moradores de rua que estavam sobre o acolhimento de abrigos. Os dados indicaram que os maiores índices de prevalência pela contaminação pelo HIV estão centrados em pessoas com práticas homossexuais, de baixa escolaridade, que possuíam sorologia positiva para sífilis e que já fizeram ou fazem uso de droga injetável. Verificou-se que os jovens apresentavam maiores probabilidades de contágio de HIV. O uso de preservativo foi pouco relatado, principalmente pelas pessoas do gênero feminino. O termo gênero representa uma construção sociocultural e especifica a maneira como a pessoa se reconhece no mundo (Butler, 2007).

Leiva-Hidalgo e Madrigal-Méndez (2013) buscaram verificar a soroprevalência da infecção por hepatite $B, C$, e vírus da Imunodeficiência Humana (HIV), determinando os fatores de risco para a presença destas infecções em grupos minoritários e de alto risco. De acordo com os autores, os hábitos praticados pelas pessoas em situação de rua do sexo masculino indicaram comportamentos de risco para a transmissão de hepatites B, C e HIV, tais como: uso de drogas injetáveis, relações sexuais sem proteção, promiscuidade, compartilhamento de itens pessoais, e tatuagens.

Esses estudos apresentam a realidade vivenciada pelas pessoas em situação de rua, no que se refere a exposição às doenças sexualmente transmissíveis, assim, é necessária uma atenção dos serviços públicos de saúde para fomentar a prevenção de doenças.

\section{CONCEPÇÕES DE SAÚDE E DOENÇA}

A pesquisa de Aguiar e Iriart (2012) analisou as práticas e sentidos relacionados a saúde e doença de pessoas em situação de rua. Os autores identificaram que as dificuldades não diferem entre as pessoas em situação de rua abrigadas e não abrigadas, porém, o segundo grupo não dispõe de acesso à alimentação, higiene, direitos sociais e ajuda terapêutica. O medo de sofrer violência física foi relatado pelos participantes, além disso, diante dos múltiplos problemas, destacou-se o uso abusivo de álcool e substâncias psicoativas, o que se intensifica devido às condições precárias de vida e a falta de acesso aos tratamentos adequados. $O$ conceito de saúde foi relacionado a sobrevivência, e os participantes abrigados associaram saúde aos cuidados com o corpo, higiene e alimentação, já a percepção de doença, tanto para os abrigados e não abrigados se referiu a incapacidade física. 
Pesquisadores investigaram o entendimento sobre saúde na percepção das pessoas em situação de rua, e verificou-se que este conceito está associado a uma condição ou circunstância não pertencente a realidade dos entrevistados: emprego, educação, alimentação, lazer, habitação, entre outros, portanto, a saúde representa um estado inatingível (Montiel, Bartholomeu, Carvalho, \& Pessoto, 2015). Para os participantes, saúde foi relacionada a vínculos familiares e afetivos, assim como, pertencer a sociedade.

É possível apresentar a hipótese de que as concepções de saúde e doença refletem a situação social vivenciada pelas pessoas em situação de rua. E em virtude da falta de acesso aos serviços básicos, a saúde representa uma utopia, assim, tal aspecto repercute nas precárias condições de vida.

\section{USO DE ÁLCOOL E DROGAS}

Panadero-Herrero e Muñoz-Lopez (2014) analisaram características das pessoas que se encontravam nas ruas há menos de 1 ano, comparando-as com aquelas que estavam no período superior há cinco anos. O consumo de álcool e drogas, maconha e heroína foram presentes entre os grupos, contudo, os participantes com mais de cinco anos nas ruas mostraram-se mais vulneráveis ao uso abusivo. O consumo de drogas ao longo de anos aumenta os fatores de risco e evidencia a ausência de qualidade de vida, um aspecto dificultador para a reinserção social.

Lima e Seidl (2015) investigaram os modos de atuação e as características do trabalho de intervenção com adultos e jovens em situação de rua, usuários de substâncias psicoativas, segundo percepções dos profissionais do consultório de rua e dos usuários. Os autores apresentaram as dificuldades enfrentadas pela equipe do consultório de rua, por exemplo: ausência de apoio dos órgãos público e privado e a exclusão desses indivíduos pela sociedade. Foram identificados os seguintes aspectos facilitadores: o trabalho multidisciplinar, a sensibilidade e vínculo de confiança entre profissionais e usuários. Na perspectiva das pessoas em situação de rua, inicialmente observou-se desconfiança no contato com a equipe, porém, ao conhecer os profissionais e o projeto, criou-se um elo de confiabilidade, o que possibilitou o acesso, assistência e atendimento às necessidades sem discriminação.

\section{MODOS DE VIDA E SIGNIFICAÇÕES}

Bachiller (2013) objetivou verificar o sentido referente a casa (ambiente doméstico) entre as pessoas que vivem nas ruas. Dentre os relatos apresentados, casa foi associada a um espaço físico no qual se sentem abrigados, seguros e confortáveis, e também representou um espaço de conflitos e tensões familiares, por outro lado, relataram que neste espaço ocorrem relações sociais fundamentais na vida do sujeito. Nos relatos de jovens e mulheres vítimas de violência doméstica, casa representou um campo de opressão injusto, de tal maneira percebem que morar na rua é a única alternativa para a busca de liberdade. 
O estudo de Costa et al. (2015) buscou compreender o cotidiano das participantes gestantes e mães de bebês com menos de dois anos, cuja gestação tivesse ocorrido nas ruas. A chegada do filho e a violência representaram fatores ansiogênicos, porém, por mais que afirmassem um desejo de oferecer melhores condições de vida à criança, a maioria não apresentou estratégias concretas para que houvesse mudanças significativas (Costa et al., 2015). As mulheres afirmaram que a situação de morar na rua representa uma forma de obter liberdade devido a ausência de regras a serem cumpridas, tendo assim, liberdade para conduzirem suas vidas. Identificou-se que o fato dessas mulheres se encontrarem em situação de rua sem documentação e/ou comprovante de residência dificulta o acesso aos serviços públicos.

A pesquisa de Rosa e Brêtas (2015) analisou as vivências de mulheres em condição de rua, e entre os motivos que levaram essas mulheres à rua foi possível identificar a violência doméstica e o uso de drogas, o que provocou rompimento do vínculo familiar e do mercado de trabalho. As mulheres relataram que a violência física é praticada por grupos e/ou comerciantes que não aceitam sua permanência na rua; e violência praticada por outras pessoas que vivem nas mesmas condições de rua por vários motivos: disputa por território, infidelidade conjugal, dívidas com traficantes, violência sexual e violência com objetivo higienista praticada por policiais.

Outro estudo buscou compreender a vivência de mulheres em situação de rua atendidas em um albergue (Biscotto, Jesus, Oliveira, \& Merighi, 2016). Foram relatadas as dificuldades para a realização de higiene pessoal e aspectos relacionados à violência física e sexual, os quais representaram os motivos para a busca de um albergue, este que representa um local que atende às necessidades básicas. O uso de álcool e o tráfico de drogas representaram fatores para a permanência dessas mulheres nas ruas, porém, identificou-se a pretensão em deixar a dependência do álcool e drogas, além disso, o desejo de ter uma casa e resgatar o convívio familiar.

As narrativas de memórias de vida, as estratégias de vida e os planos futuros de pessoas em situação de rua foram investigados (Andrade, Costa, \& Marquetti, 2014). Os entrevistados afirmaram que a rua possui um imã que simboliza a liberdade e isso representou a justificativa para a situação de rua, pois nas ruas não existem regras, e outra justificativa foi o rompimento de vínculos com a família. $\mathrm{E}$ referente à renda e trabalho, realizam os seguintes serviços: cuidador de carros, catador de recicláveis e na montagem de barracas na praia. Relataram a violência sofrida pelos agentes de segurança pública, o que reflete a negligência de direitos humanos. Outro ponto foi a dificuldade para conseguir alimentos, tendo que pedir em comércios ou procurar em lixeiras. Alguns desejam sair da situação de rua, buscar um emprego e ter outras condições de vida, mas outros revelam a intenção de continuar nas ruas com trabalho informal. 
Outros autores objetivaram analisar os modos de vida da população em situação de rua (Kunz, Heckert, \& Carvalho, 2014). De acordo com a pesquisa, os participantes utilizam-se de histórias comoventes para conseguir dinheiro, ou então prestam algum serviço para comerciantes. A coleta de materiais recicláveis representou a principal atividade, e referente a alimentação apresentaram as dificuldades para conseguir acesso aos itens básicos para a sobrevivência diária. Sobre defesa e segurança nas ruas, identificou-se relatos de violência por parte de agentes de segurança e o uso de facas para a defesa pessoal. A higiene pessoal diária é realizada em espaços públicos ou em casas de pessoas que oferecem ajuda, e para diversão desenvolvem encontros com seus grupos para conversar e compartilhar suas histórias.

Verificou-se que vários fatores levaram pessoas em situação de rua à exclusão do mercado de trabalho, por exemplo, idade avançada, aposentadoria precoce, falta de qualificação, e problemas de saúde (Henrique, Santos, \& Vianna, 2013). Nesta pesquisa, as pessoas em situação de rua atribuíram ao trabalho o significado de vida digna, auto realização e também uma forma de obter saúde, seja física ou mental.

Outro estudo buscou conhecer os modos de vida e os motivos que levaram as pessoas à situação de rua (Donoso, Bastos, Faria, \& Costa, 2013). O rompimento do vínculo familiar e o uso de drogas foram os principais motivos. Exercem trabalhos de catadores de materiais recicláveis e ficam pedindo esmolas. Sobre as expectativas para o futuro, alguns revelaram o desejo de tratamento de vício em drogas, buscar um emprego formal ou voltar para a terra natal, e outros manifestaram o desejo de permanecer nas ruas em virtude da liberdade. A respeito da auto percepção, se percebem excluídos da sociedade e com vergonha de sua situação. Na pesquisa de Matias (2011), a insegurança dos jovens foi algo identificado, bem como a hostilidade do ambiente em que estão inseridos. Identificou-se receio por parte dos moradores próximos a locais onde esses jovens se encontravam, apesar de afirmarem considerar esses jovens seres humanos sem diferenciação.

Os modos de vida e as significações atribuídas às experiências vivenciadas incluem aspectos de violência, uso de drogas, discriminação e rupturas de vínculos familiares. Neste sentido, existem inúmeros problemas que representam desafios a serem enfrentados pelas equipes da assistência social e saúde. Assim, promover a reinserção social representa uma estratégia que envolve diferentes setores e, principalmente, o envolvimento das pessoas em situação de rua na luta diária dos problemas e vulnerabilidades.

\section{IDENTIDADE SOCIAL}

A análise de narrativas de oito pessoas em situação de rua do gênero masculino possibilitou identificar que no contexto familiar os entrevistados relataram os problemas relacionados ao consumo de álcool e a violência, os quais representaram os principais motivos que levaram à situação de rua; e referente a 
rua, contexto principal, alguns vivem desde a infância devido a violência vivenciada no ambiente familiar, e outros iniciaram a moradia na rua em idade adulta devido o envolvimento com álcool e outras drogas (Flores, Contreras, Hernández, Levicoi, \& Vargas, 2015).

A identidade social foi observada por meio das ações realizadas pelos mesmos: pedir dinheiro e uso de álcool e drogas (Flore et al., 2015). Para os autores, a construção da identidade social ocorre por meio das condutas nas interações para que se possa integrar um grupo social. Essas pessoas encontram nas ruas seus pares e se identificam com outros moradores, assim, ocorre o compartilhamento do espaço físico, de objetivos e interesses. Com isso se percebem pertencentes ao grupo e muitas vezes não conseguem deixá-lo, pelo fato de que construíram identidade social.

Matias (2013) buscou compreender as narrativas de histórias de vidas de jovens em situação de rua, e a pesquisa foi realizada com o objetivo de investigar os processos de construção das identidades. Para a apresentação dos resultados foram escolhidas duas narrativas, sendo uma do gênero masculino e outra do feminino, a fim de expor as diferentes subjetividades encontradas na pesquisa. $\mathrm{Na}$ primeira narrativa, de um jovem com 17 anos do gênero masculino, verificou-se que o mesmo se coloca como responsável por estar em situação de rua, pelo fato de ter tido o interesse em conhecer esse espaço, e a partir disso ocorreu o afastamento do vínculo familiar. Já na segunda narrativa, representada por uma jovem de 16 anos do gênero feminino, notou-se que ela não se responsabiliza pela situação que se encontra, devido a ocorrência de processos anteriores que a levaram a viver na rua. Os fatores que mantêm esses jovens em situação de rua foram os seguintes: o envolvimento com as drogas, prostituição, abandono, e a interrupção do vínculo familiar.

Uma pesquisa buscou compreender a exclusão social e as transformações nas identidades sociais em adultos em situação de rua (Alcantara, Abreu, \& Farias, 2015). Nesta pesquisa de estudo de caso participaram duas pessoas do sexo masculino que se encontravam numa casa de acolhimento. Foram identificadas representações sociais negativas acerca de pessoas em situação de rua, sendo estas vistas como miseráveis, excluídos e invisíveis perante a sociedade. Para os autores, a exclusão social é geradora de culpa entre as pessoas em situação de rua, o que repercute diretamente na formação de sua identidade. Nos relatos verificouse que o uso de álcool e drogas representou a única forma de enfrentar a situação de vulnerabilidade. Após análise biográfica de um dos participantes, não foram identificados vínculos familiares e afetivos, mas observou-se que ter um emprego representa algo importante na construção de sua identidade, pois traz consigo reconhecimento e valorização social. E referente ao segundo participante, verificou-se relações familiares sólidas, o convívio com grupos na casa de acolhida representou uma forma de ressignificar sua trajetória de vida. 
Os estudos sobre identidade social de pessoas em situação de rua apresentam a perspectiva de que as vivências cotidianas são geradoras de identidades marcadas por processos de exclusão. Verificou-se que as ações realizadas no cotidiano buscam construir a noção de pertencimento ao grupo, mas por outro lado, a manutenção de hábitos referentes ao uso de drogas inviabiliza a emancipação destas pessoas. Assim, é necessário desenvolver trabalhos em grupo no contexto em que estão inseridos para promover novas perspectivas de vida.

\section{DISCUSSÃO}

Nos estudos sobre aspectos sociodemográficos, verificou-se o aumento da população em situação de rua de adultos. Em outro estudo, Parro (2015) comparou os dados censitários dos anos de 2000, 2009 e 2011, e identificou o aumento da população em situação de rua em São Paulo, de 8.706 para 15.905 entre os anos de 2000 e 2015. Estes dados corroboram o que foi identificado nesta revisão sistemática referente o crescente número de pessoas em situação de rua, mas se faz necessário compreender as especificidades desta população relacionadas à gênero e idade.

Os resultados referentes à categoria processo saúde e doença possibilitaram compreender aspectos específicos, por exemplo, os estudos relacionados aos transtornos mentais e de personalidade evidenciaram lacunas nos tratamentos e atendimentos especializados. De acordo com Borysow e Furtado (2013), o transtorno mental representa um aspecto agravante, pelo fato de que as vivências na rua poderão repercutir no surgimento de novas patologias ou maximizar aquelas existentes.

No que se refere à vulnerabilidade ao HIV e outras doenças sexualmente transmissíveis, as pesquisas identificaram comportamentos de risco entre as pessoas em situação de rua. Santana (2014) ressalta que ações que buscam melhorias das condições mentais dos moradores de rua são tão importantes quanto à promoção da saúde e prevenção de doenças. Logo, percebe-se a necessidade de fomentar projetos de promoção da saúde e auxiliar os profissionais de saúde para atuação com a população em situação de rua, ao considerar a vulnerabilidade social e os fatores de proteção e de risco. Para Hallais e Barros (2015), é fundamental priorizar práticas de saúde que sejam efetivas e abrangentes, as quais respeitem a individualidade e o contexto de cada pessoa. É necessário uma análise crítica e relativizada para evitar associar infecção do vírus HIV à população de homossexuais, pelo fato de que isto pode maximizar os preconceitos e estigmas sociais geradores de exclusão. Assim, recomenda-se a realização de novos estudos, com uma visão ampliada referente a esta temática buscando direcionar as estratégias de promoção da saúde para a população geral.

E diante da complexidade do atendimento de saúde para as pessoas em situação de rua, o Ministério da Saúde criou em 2011 o consultório na rua cujo objetivo é ampliar a qualidade da atenção integral à saúde, o que possibilita o acesso pautado 
nos princípios da integralidade com uma equipe multidisciplinar especializada para atender as demandas e necessidades de saúde (Ministério da Saúde, 2012). No entanto, percebe-se na prática que mesmo com a criação do Consultório na rua, os atendimentos precisam de aprimoramento técnico em virtude dos desafios para minimizar o uso abusivo de álcool e outras drogas.

Com relação às concepções de saúde e doença, foi possível compreender diferentes sentidos atribuídos pelas pessoas em situação de rua, por exemplo, saúde relacionada à ausência de doença física ou psíquica; dispor de um elo afetivo com a família, amigos, sentir-se parte da sociedade e possuir meios que garantam a subsistência: alimentação, trabalho, moradia, lazer entre outros. Conforme a Lei 8.080, a saúde é o conjunto de ações individuais e coletivas que buscam promover qualidade de vida por meio da valorização da integralidade, que se refere aos modos de viver, as relações entre o indivíduo e o social, e o acesso aos serviços básicos referentes à alimentação, moradia, segurança pública, e educação (Presidência da República, 1990). Neste sentido, as pessoas em situação de rua têm o direito de reivindicar o acesso aos serviços básicos para promover saúde e qualidade de vida.

E sobre o uso de álcool e drogas, verificou-se que a dependência química representa um aspecto comum entre as pessoas em situação de rua. Para Pacheco (2014), o uso de álcool e de substâncias psicoativas contribui para maximizar o processo de exclusão social desses indivíduos. O consumo de álcool e drogas representa uma prática frequente e um fator agravante para que as pessoas se mantenham vivendo em situação de rua, de tal maneira que se torna uma problemática no cenário da saúde pública (Silva, Frazão, \& Linhares, 2014). Deste modo, verifica-se a relevância de políticas públicas que visam reduzir o consumo de álcool e de substâncias psicoativas por meio da redução de danos, para que seja possível oferecer acolhimento fundamentado na integralidade do cuidado.

Ao analisar os resultados obtidos nas pesquisas relacionadas aos modos de vida e significações, verificou-se pontos comuns referentes à violência, liberdade encontrada na rua, fragilização de vínculos familiares e uso de drogas. Os vínculos familiares se tornam frágeis e na maioria dos casos isso ocorre devido ao uso abusivo de álcool e drogas (Reis \& Azevêdo, 2019; Arruda, Oliveira, \& Almeida, 2015). Nas pesquisas identificadas nesta revisão sistemática, os resultados foram semelhantes as informações apresentadas em outros estudos (Arruda et al., 2015; Reis \& Azevêdo, 2019), entretanto, é importante valorizar a singularidade de cada indivíduo, a história de vida e seus relatos, para contextualizar os motivos que levaram as pessoas à situação de rua.

Os indivíduos que se encontram em situação de rua sofrem constantes violências e humilhações de outras pessoas e até mesmo de profissionais de serviços públicos, e a partir disso, inicia-se uma dinâmica recorrente de opressão (Moura, Ximenes, \& Sarriera, 2013). As pessoas em situação de rua apresentam o desejo de retornarem à família (Cociuffo, 2013), o que foi encontrado nos estudos desta 
revisão sistemática, mas por outro lado, não existem ações claras para o retorno ao ambiente familiar, assim, continuam vivendo nas ruas em virtude da sensação de liberdade. É possível compreender que o desejo de retornar ao ambiente familiar está no plano da ideação, pois não existem estratégias ou ações objetivas, por exemplo, há uma falta de estabelecimento de contatos com a família.

A partir da análise realizada sobre a identidade social das pessoas que se encontram em situação de rua, observou-se que os estudos voltados à essa temática tiveram como principais objetivos, compreender as narrativas, investigar os processos de construção das identidades, e compreender a exclusão social e suas repercussões na construção da identidade. Verificou-se que o processo de construção da identidade social é estruturado por meio de conflitos presentes no contexto familiar, e diante da ocorrência do uso abusivo de substâncias psicoativas que permitem ao indivíduo a socialização em um novo grupo social, no qual o mesmo se identifica e isso gera sentimento e pertencimento.

Segundo Araújo e Tavares (2015), a identidade social é representada de diversas maneiras na sociedade, dependendo do contexto em que cada grupo está inserido, gerando assim diferentes sentimentos de pertença. Para as pessoas que se encontram em situação de rua, a percepção e estruturação de suas identidades ocorrem por meio do compartilhamento de suas histórias de vida e identificação com aqueles que convivem diariamente. A identificação com outras pessoas e o desenvolvimento de redes de apoio na comunidade foi um aspecto observado nas pesquisas, o que apresenta congruência com os resultados de Araújo e Tavares (2015). É necessário ressaltar que, a identidade social da pessoa que se encontra em situação de rua e utiliza drogas, muitas vezes é julgada, pelo fato de que há um olhar negativo da sociedade para essa população (Moura \& Ximenes, 2016).

Para Moura e Ximenes (2016), as pessoas em situação de rua que utilizam substâncias psicoativas podem passar por uma modificação em suas relações familiares diante da falta de aceitação, o que repercute, em alguns casos, na construção de novos vínculos afetivos no grupo social. Portanto, na rua essas pessoas tendem a receber apoio que não tiveram no ambiente familiar e social, sendo acolhidos sem julgamentos. Contudo, entende-se que o processo de construção da identidade social, inclui a identificação e o sentimento de pertença dos mesmos ao grupo, e também o olhar do social para esses moradores de rua, o qual ocorre de maneira negativa e gera exclusão social.

\section{CONSIDERAÇÕES FINAIS}

A finalidade do presente estudo foi realizar uma revisão sistemática das pesquisas empíricas referentes às pessoas em situação de rua. Verificou-se o crescimento estatístico desta população, o que representa um fator agravante para vulnerabilidade social em virtude das características deste perfil populacional: o uso abusivo de álcool e drogas, as violências vivenciadas, e a fragilização e rompimento de vínculos familiares. 
Destacam-se os estudos sobre o processo saúde e doença, os quais indicam a necessidade de cuidados e atenção integral, assim como, o funcionamento adequado dos serviços públicos de saúde. Sugere-se a ampliação dos programas de saúde coletiva com o objetivo de promover saúde, além do acompanhamento destas pessoas por meio de uma equipe multidisciplinar com profissionais capacitados para oferecer acolhimento e cuidado integral.

Os resultados apresentados evidenciam a necessidade de minimizar a exclusão social por meio do desenvolvimento e divulgação de programas do governo que possibilitem a resolutividade nas situações de risco, isto permite promover a ampliação dos serviços para atender as demandas psicossociais. Os Centros Pops representam uma estratégia da Política de Assistência Social, assim, para atingir a resolutividade é necessário desenvolver ações intersetoriais. O objetivo desta revisão sistemática foi atingido, o que evidenciou maior número de publicações em revistas nacionais com adultos do gênero masculino. Dessa forma, ressalta-se a importância de futuras pesquisas nas quais sejam incluídas crianças e adolescentes, visto que fazem parte desse cenário. Logo, é possível sugerir a necessidade de estudos com intervenções voltadas para o processo saúde e doença, para que sejam capazes de viabilizar o acesso aos serviços e políticas públicas.

\section{REFERÊNCIAS}

Aguiar, M. M., \& Iriart, J. A. B. (2012). Significados e práticas de saúde e doença entre a população em situação de rua em Salvador, Bahia, Brasil. Cadernos de Saúde Pública, 28(1), 115-124. doi:10.1590/S0102-311X2012000100012.

Alcantara, S. C., Abreu, D. P., \& Farias, A. A. (2015). Pessoas em situação de rua: Das trajetórias de exclusão social aos processos emancipatórios de formação de consciência, identidade e sentimento de pertença. Revista Colombiana de Psicologia, 24(1), 129-143. doi:10.15446/rcp.v24n1.40659.

Andrade, L. P., Costa, S. L., \& Marquetti, F. C. (2014). A rua tem um ímã, acho que é a liberdade: Potência, sofrimento e estratégias de vida entre moradores de rua na cidade de Santos, no litoral do Estado de São Paulo. Saúde e Sociedade, 23(4), 1248-1261. doi:10.1590/S010412902014000400011.

Araújo, P. T., \& Tavares, M. G. (2015). População em situação de rua: Identidade social e a dialética da inclusão/exclusão. Ciências humanas e sociais, 2(3), 113-131.

Arruda, M. A, Oliveira, C. H, \& Almeida, L. P. (2015). A história de vida de pessoas em situação de rua na cidade de Campo Grande/MS - Brasil. DIRE, 6, 37-47. doi:10.25965/dire.583

Bachiller, S. (2013). Una análisis etnográfico sobre las personas en situación de calle y los sentidos de hogar. Sociedade e Cultura, 16(1), 81-90.

Barata, R. B., Carneiro-Júnior, N., Ribeiro, M. C. S. A., \& Silveira, C. (2015). Desigualdade social em saúde na população em situação de rua na cidade de São Paulo. Saúde e Sociedade, 24(Suppl. 1), 219-232. doi: 10.1590/S0104-12902015S01019.

Bardin, L. (2002). Análise de conteúdo. Lisboa: Edições.

Bireme (2017). Descritores em Ciências da Saúde: DECS. Recuperado de: http://decs.bvsalud.org

Biscotto, P. R., Pinto de Jesus, M. C., Silva, M. H., Oliveira, D. M., \& Merighi, M. A. B. (2016). Compreensão da vivência de mulheres em situação de rua. Jornal da Escola de Enfermagem da USP, 5Q(5), 750-756. doi: 10.1590/s0080-623420160000600006

Borysow, I. C., \& Furtado, J. P. (2013). Acesso e intersetorialidade: O acompanhamento de pessoas em situação de rua com transtorno mental grave. Physis: Revista de Saúde Coletiva, 23(1), 3350. doi:10.1590/S0103-73312013000100003.

Butler, J. (2007). El feminismo e la subversión de la identidad. Barcelona: Paidós. 
Cociuffo, T. (2013). O ensino de Psicopatologia no cotidiano das ruas de São Paulo: Um relato de experiência. Psicologia em Revista, 22(2), 251-260.

Costa, S. L., Vida, C. P. C., Gama, I. A., Locatelli, N. T., Karam, B. J., Ping, C. T., ... Bernardes, A. F. M. (2015). Gestantes em situação de rua no município de Santos, SP: Reflexões e desafios para as políticas públicas. Saúde e Sociedade, 24(3), 1089-1102. doi:10.1590/S010412902015134769.

Donoso, M. T. V., Bastos, M. A. R., Faria, C. R., \& Costa, A. A. (2013). Estudo etnográfico sobre pessoas em situação de rua em um grande centro urbano. REME Revista Mineira de Enfermagem, 17(4), 894-901. doi:10.5935/1415-2762.20130065.

Farias, D. C. S., Rodrigues, I. L. A., Nogueira, L. M. V., \& Marinho, I. C. (2014). Saberes sobre saúde entre pessoas vivendo em situação de rua. Psicologia e Saber Social, 3(1), 70-82. doi:10.12957/psi.saber.soc.2014.10064.

Ferreira, F. P. M. (2011). Crianças e adolescentes em situação de rua e seus macro determinantes. Saúde e Sociedade, 20(2), 338-349. doi:10.1590/S0104-12902011000200007

Flores, M., Contreras, C., Hernández, Y., Levicoi, Y., \& Vargas, C. (2015). Ocupación e identidade social en personas en situación de calle de la ciudad de Punta Arenas. Revista Chilena de Terapia Ocupacional, 15(2). doi: 10.5354/0719-5346.2015.38159

Grangeiro, A., Holcman, M. M., Onaga, E. T., Alencar, H. D. R., Placco, A. L. N., \& Teixeira, P. R. (2012). Prevalência e vulnerabilidade à infecção pelo HIV de moradores de rua em São Paulo, SP. Revista de Saúde Pública, 46(4), 674-684. doi:10.1590/S0034-89102012005000037.

Gusmão, B. S., Leite, K. L. Y., Monteiro, L., Umeno, M. B., Pessutti, M. S., Santos, ... Falcão, D. V. (2012). Idoso em situação de rua e vivência em centros de acolhida: Uma revisão de literatura. Revista Temática Kairós Gerontologia, 15(6), 313-331.

Hallais, J. A. S., \& Barros, N. F. (2015). Consultório na Rua: visibilidades, invisibilidades e hipervisibilidade. Cadernos de Saúde Pública, 31(7), 1497-1504. doi:10.1590/0102$311 X 00143114$.

Henrique, R. A., Santos, C. M., \& Vianna, J. J. B. (2013). Sentidos e significados do trabalho entre pessoas em situação de rua. Psicologia para América Latina, 24, 109-120.

Herrera N. C., Rojas A. C., \& Jiménez, C. L. (2016). Prevalência de HIV em crianças e adolescentes vivendo na rua e sujeitos a exploração sexual comercial: Uma revisão sistemática. Cadernos de Saúde Pública, 32(10), 1-13. doi:10.1590/0102-311X00134315.

Instituto de Pesquisa Econômica Aplicada (2016). Estimativa da População em Situação de rua no Brasil. Brasília: DF.

Kunz, G. S., Heckert, \& Carvalho, A. L. (2014). Modos de vida da população em situação de rua: Inventando táticas nas ruas de Vitória/ES. Fractal: Revista de Psicologia, 26(3), 919-942. doi:10.1590/1984-0292/1192.

Leiva-Hidalgo, J., Madrigal-Méndez, A., \& Salas-Segura, D. (2013). Seroprevalencia de hepatitis B, C y VIH en indigentes en Costa Rica. Revista Costar Salud Pública, 22(2), 113-118.

Lima, H. S., \& Seidl, E. M. F. (2015). Consultório na rua: Atenção a pessoas em uso de substâncias psicoativas. Psicologia em Estudo, 20(1), 57-69. doi:10.4025/psicolestud.v20i1.24697.

Matias, H. J. D. (2011). Jovens em situação de rua: Espaço, tempo, negociações de sentido. Psicologia \& Sociedade, 23(2), 237-247. doi:10.1590/S0102-71822011000200004.

Matias, H. J. D. (2013). Sedução e descaminho: Narrativas e identidades de jovens em situação de rua. Psicologia: Reflexão e Crítica, 26(3), 543-551. doi:10.1590/S0102-79722013000300014.

Ministério da Saúde (2008). Política nacional para inclusão social da população em situação de rua. Brasília:DF. Recuperado de http://www.planalto.gov.br/ccivil_03/_Ato20072010/2009/Decreto/D7053.htm.

Ministério da Saúde (2012). Manual sobre o cuidado a saúde junto à população em situação de rua. Departamento de Atenção Básica. Brasília: DF. Recuperado de http://dab.saude.gov.br/portaldab/biblioteca.php?conteudo=publicacoes/manual_saude_p opulacao_rua

Ministério da Saúde (2014). Saúde da população em situação de rua: Um direito humano. Brasília, DF: Recuperado

http://bvsms.saude.gov.br/bvs/publicacoes/saude_populacao_situacao_rua.pdf

Ministério do Desenvolvimento Social e Combate à Fome (2014). Orientações técnicas: Centro de referência especializado para população em situação de rua e serviço especializado para pessoas em situação de rua. Brasília, DF. 
Ministério do Planejamento e Gestão (2016). Estimativa da população em situação de rua no Brasil. Brasília, DF.

Montiel, J. M., Bartholomeu, D., Carvalho, L. F., \& Pessotto, F. (2015). Avaliação de transtornos da personalidade em moradores de rua. Psicologia: Ciência e Profissão, 35(2), 488-502. doi:10.1590/1982-370301992013

Moura, J. F., \& Ximenes, V. M. (2016). O lugar do uso de drogas na identidade de uma pessoa em situação de rua. Gerais: Revista Interinstitucional de Psicologia, 9(2), 259-276.

Moura, J. F., Ximenes, V. M., \& Sarriera, J. C. (2013). Práticas de discriminação às pessoas em situação de rua: Histórias de vergonha, de humilhação e de violência em Fortaleza, Brasil. Revista de Psicologia, 22(2), 18-28. doi:10.5354/0719-0581.2014.30850

Pacheco, M. E. A. G. (2014). Políticas públicas e capital social: O projeto consultório de rua. Fractal: Revista de Psicologia, 26(1), 43-58. doi:10.1590/S1984-02922014000100005.

Panadero-Herrero, S., \& Muñoz-López, M. (2014). Salud, calidade de vida y consumo de sustâncias em función del tempo en situación sin hogar. Anales de psicología, 3a(1), 70-77. doi:10.6018/analesps.30.1.137911.

Parro, E. A., \& Adamos, R. E. (2015). Pesquisa censitária da população em situação de rua, caracterização socioeconômica da população adulta em situação de rua e relatório temático de identificação das necessidades desta população na cidade de São Paulo. Fundação Instituto de Pesquisas Econômicas.

Presidência da República. Casa Civil. Subchefia para Assuntos Jurídicos. (1990). Lei no 8.080, de 19 de setembro de 1990: Dispõe sobre as condições para a promoção, proteção e recuperação da saúde, a organização e o funcionamento dos serviços correspondentes e dá outras providências. Brasília, DF. Recuperado em 23 de Novembro de 2017. Disponível em: http://conselho.saude.gov.br/legislacao/lei8080_190990.htm

Presidência da República. Casa Civil. Subchefia para Assuntos Jurídicos. (2009). Decreto $n^{\circ} 7.053$, de 23 de dezembro de 2009: Institui a Política Nacional para a População em Situação de Rua e seu Comitê Intersetorial de Acompanhamento e Monitoramento, e dá outras providências. Brasília, DF. Disponível em http://www.planalto.gov.br/ccivil 03/ ato20072010/2009/decreto/d7053.htm

Reis, T. C. M., \& Azevêdo, A. V. S. (2019). Vivências de homens em situação de rua no sul do Brasil. Contextos Clínicos, 12(3), 976-999. doi: 10.4013/ctc.2019.123.13

Risco, M. M., Léon, E., Dongo, M., \& Munayco, C. V. (2015). Características sociodemográficas y de salud de los adultos mayores en situación de calle en Lima, Perú. Revista Peruana de Medicina Experimental y Salud Publica, 32(4), 693-700.

Rosa, A. S., \& Brêtas, A. C. P. (2015). A violência na vida de mulheres em situação de rua na cidade de São Paulo, Brasil. Interface - Comunicação, Saúde, Educação, 19(53), 275-285. doi:10.1590/1807-57622014.0221

Salavera, C. (2014). Influência de los transtornos mentales y de la personalidade nel abandono de processos en personas sin hogar. Anales de psicología, 3a(1), 78-82. doi:10.6018/analesps.30.1.124081.

Sampaio, R. F., \& Mancini, M. C. (2007). Estudos de revisão sistemática: Um guia para síntese criteriosa da evidência científica. Revista Brasileira de Fisioterapia, 11(1),83-89. doi: 10.1590/S141335552007000100013.

Santana, C. (2014). Consultórios de rua ou na rua? Reflexões sobre políticas de abordagem à saúde da população de rua. Cadernos de Saúde Pública, 3018), 1798-1799. doi:10.1590/0102311XCA010814

Scappaticci, A. L. S. S., \& Blay, S. L. (2010). Mães adolescentes em situação de rua: Uma revisão sistemática da literatura. Revista de Psiquiatria do Rio Grande do Sul, 32(1), 3-15.

Silva, F. P., Frazão, I. S., \& Linhares, F. M. P. (2014). Práticas de saúde das equipes dos consultórios de rua. Cadernos de Saúde Pública, 30(4), 805-814. doi:10.1590/0102-311X00100513.

\section{CONFLTTOS DE INTERESSES}

Não há conflitos de interesse. 


\section{SOBRE OS AUTORES}

Gesyele Batista Oliveira é Psicóloga pela Universidade Tuiuti do Paraná.

E-mail: eleyseg@gmail.com

1. https://orcid.org/0000-0002-0261-436X

Aline Moraes Selusnhaki é Psicóloga pela Universidade Tuiuti do Paraná e Pós-Graduanda em Comportamento Alimentar na Pós FG.

E-mail: aline.moraess@hotmail.com

(1) https://orcid.org/0000-0003-3502-4451

Débora Mayumi Higa é Psicóloga pela Universidade Tuiuti do Paraná.

E-mail: deborahiga@gmail.com

(1) https://orcid.org/0000-0002-9174-9505

Jackeline Claudino Sousa Araujo é Psicóloga pela Universidade Tuiuti do Paraná e Pós-Graduanda em Psicologia da Saúde e Hospitalar na Faculdades Pequeno Príncipe

E-mail: psicojackelinearaujo@hotmail.com

(1) https://orcid.org/0000-0002-5267-2066

Tomás Collodel Magalhães Reis é Psicólogo e Mestre em Psicologia pela Universidade Tuiuti do Paraná.

E-mail: tomreis@gmail.com

(1) https://orcid.org/0000-0001-6384-5543

Adriano Valério dos Santos Azevêdo é Docente do Programa de Pós-Graduação em Psicologia da Universidade Tuiuti do Paraná

E-mail: adrianoazevedopsi@yahoo.com

(1) https://orcid.org/0000-0003-0238-3423 